\title{
OBSERVATIONAL PROBLEMS IN EXTRAGALACTIC ASTRONOMY
}

\author{
HALTON ARP \\ Max-Planck-Institut für Astrophysik \\ 8046 Garching bei München, Germany
}

\begin{abstract}
A large number of observations have accumulated which represent baffling mysteries from the standpoint of current astronomy. A sample of the most crucial of these cases is reviewed and updated. Evidence has slowly built up to the point where often each individual object is a formidable challenge to conventional theory. But, most importantly, it is now possible to appreciate the common pattern which mutually reinforces all the separate results.

These empirical discordances point directly at known weaknesses in the Big Bang theory of the origin of the universe. New data is used to introduce a proposal which accounts for both the data which gave rise to the Big Bang interpretation and which also satisfies the discordant results which have accumulated over the last quarter of a century. Though perhaps only a primitive beginning, the new interpretation demonstrates that observations need not be discarded because they disagree with current theory. The major conclusion from the cases discussed here is that the present observational paradoxes represent spectacular opportunities to make new and fundamental discoveries about the universe.
\end{abstract}

\section{Opportunities to Discover New Information}

Many people view problems as textbook exercises to be worked on until they got the right answer. But in astronomy we generally do not know answers so observational discordances are in reality opportunities to learn something new about how the universe actually works. For about 25 years $I$ have been trying to publish information on objects which appear to contradict current theory. Today I would like to look again at some of the most disturbing of these objects and summarize them using the newest data. With the plethora of powerful new telescopes and detectors these provocative problems represent fundamental understanding of the universe waiting to be harvested. Both astronomers currently engaged in research and young astronomers starting an observational career, I feel, would achieve outstanding results from further observation of these objects. At the end we will see that perhaps different connections between observational facts may be required - and thus we may gain further theoretical insight into underlying laws of nature.

\section{Weaknesses In Current Theory}

It is clear that the ultimate authority is observational and that it is necessary to argue empirically and inductively from the data. Nevertheless it is helpful to know where other evidence has already cast doubt on the current paradigm. A brief mention of current theoretical difficulties will be useful in giving perspective to the observational problems to be discussed. 


\subsection{THE SMOOTHNESS OF THE COSMIC BACKGROUND RADIATION}

The pervasive background radiation of $2.7^{\circ} \mathrm{K}$ temperature is commonly held to be the strongest proof of the Big Bang. This is not my field of expertise but $\mathrm{I}$ have listened to specialist say "The background radiation should show small scale variations due to formation of galaxies early in the Big Bang. But the microwaves are observed to be smooth to a startling degree. This is very worrying and if it gets smoother we will be in serious trouble." Then it is measured to be smoother and the statement is repeated over again. The upshot is that many have concluded that what was once the strongest argument for the Big Bang has now become evidence against it.

\subsection{THE AGE OF THE OLDEST STARS VERSUS THE EXPANSION AGE OF THE UNI- VERSE}

A fundamental calculation which links atomic cross section date from nuclear physics laboratories with stellar structure theories of astrophysics enables the evolutionary ages of stars to be calculated. The ages of the oldest stars have thus been measured with increasing accuracy for more than 30 years in globular clusters. The current result is $14<$ age $<17$ yrs. The expansion age of the universe depends on its rate of expansion as measured by the Hubble constant (in $\mathrm{km} \mathrm{s}^{-1} \mathrm{Mpc}^{-1}$ ). But almost all measures of the Hubble constant currently give straightforward estimates of the age of the universe which are shorter than the age of the oldest stars.[1] Complications must be added to avoid this paradox. Quoting van den Bergh [2]: "...the universe must either be open or, if it is closed, must have a non-zero value of the cosmological constant."

\subsection{THE EXISTENCE OF YOUNG GALAXIES}

The key cosmogonic requirement of the Big Bang is that the universe originated at a single point in space-time and all the galaxies in it formed shortly afterwards. This operational definition of the Big Bang is precisely where one can test the hypothesis. The crucial question is simply: Are all galaxies old? All galaxies were supposed to have been formed $\sim 15 \times 10^{9}$ years ago. But it is readily apparent to even casual observers that we see many galaxies filled with young, blue stars (whose ages are predominantly in the range $10^{6}-10^{8}$ years). The obvious conclusion that these galaxies were recently created was dismissed long ago with the argument that all galaxies formed about the same epoch in the early universe but that some have been forming stars more slowly than others.

I would like to point to evidence which refutes this key requirement of the Big Bang. It is simply that if galaxies all formed $\sim 15 \times 10^{9}$ years ago, and that star formation proceeded more slowly in some, that today we should see galaxies in all states of apparent youth, including many galaxy sized masses of hydrogen gas that have not yet started forming stars. We do not.

Galaxy sized masses of hydrogen are easily detectable out to considerable distances in the universe, particularly with sensitive radio telescopes. Nearby, at intermediate redshifts and at redshift $z=4$ where galaxy formation should be very active on the Big Bang hypotheses, galaxy sized clouds of hydrogen are not found. (for references see [3])

In essence the argument is that if galaxy sized hydrogen clouds were all born $\sim$ $15 \times 10^{9}$ years ago that they would have had to, one by one, flare up in episodes of star formation between that epoch and the present time. But this peculiar behavior would have had to suddenly stop at just our particular time in the universe at which we are observing. (Since there are no more proto galaxy sized clouds.) This is so unlikely a circumstance as to, in my opinion, constitute a disproof of Big Bang.

\section{The Young Quasar/Galaxy $3 \mathrm{C} 120$}

Do young galaxies look as if they were ancient gas clouds suddenly now condensing stars 
throughout their volume? On the contrary - they are characteristically centered on a small, energetic core out of which most of the material in the galaxy appears to flow. One example is $3 \mathrm{C} 120$, a blue compact galaxy originally classified as a quasar because of its star like appearance. It has optical jets, young stars [4] and extensive radio jets all emanating from its condensed, active nucleus.
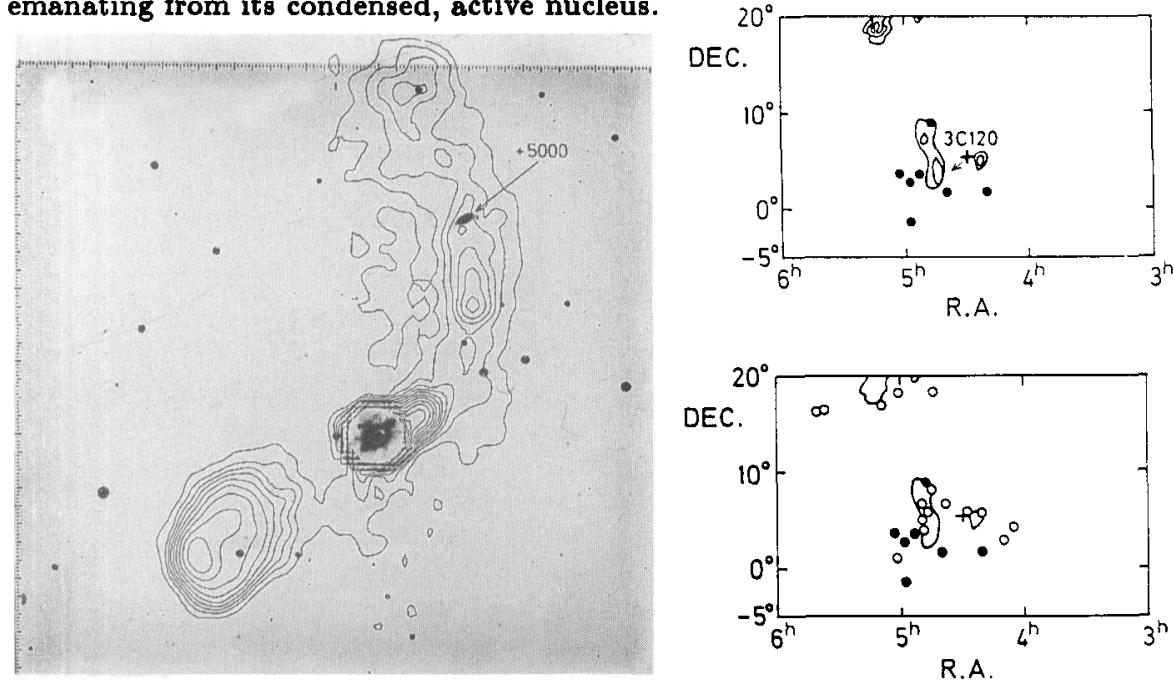

Fig. 1 Radio isophotes superposed

Fig. 2 Concentration of objects around on photograph of $3 \mathrm{C120.}$

3C120 (cross). Filled circles are quasars.

Fig. 1 is a good illustration of the general tendency for galaxies to eject radio material in roughly opposite directions. Luminous matter in the form of optical jets is also often observed in the direction of these radio ejections. Note the extremely peculiar galaxy lying in the northwest radio extension. It has a redshift $\sim 5,000 \mathrm{~km} \mathrm{~s}^{-1}$ higher than the $\sim 10,000 \mathrm{~km} \mathrm{~s}^{-1}$ redshift of $3 \mathrm{C} 120$ itself.

Now note in Fig. 2 what happens when the area around $3 \mathrm{C} 120$ is searched. The filled circles represent all radio quasars $\left(S_{6} \geq 0.3\right)$ with redshift $z \geq 1.35$. They are roughly in the direction of the $\mathrm{SE}$ lobe of the radio ejection from $3 \mathrm{C} 12 \overline{0}$. Another peculiar kind of object is the low surface brightness galaxies with $4500<c z_{0}<5300 \mathrm{~km} \mathrm{~s}^{-1}$ (open circles). Finally the open contours show $\sim 0$ redshifted hydrogen clouds within the pictured area, two of which essentially bracket $3 \mathrm{C} 120$.

As noted in reference [5] "...the concentration of these objects in the vicinity of this unique, active galaxy has a negligible chance of being accidental and that all those objects of diverse redshift are at the same nearby distance. This smaller distance reduces the supposed superluminal motions in 3C120 to quite precedented ejection velocities."

The strangeness of this conclusion is only matched by the improbability of its being accidental. It is therefore of the utmost importance to see whether further, independent cases show a similar pattern of activity and association.

\section{The Virgo Cluster and the Origin of Quasars}

This story begins in 1966 when my Atlas of Peculiar Galaxies was finished. Checking for radio identifications with the newly catalogued objects I surprisingly discovered radio sources paired across the disturbed galaxies. Obviously they had been ejected out in opposite directions by explosions in the nucleus. But among these radio sources were some quasars with high redshifts. So these quasars could not be at the enormous distance their redshift indicated - but instead had to be at the relatively close distance of the 


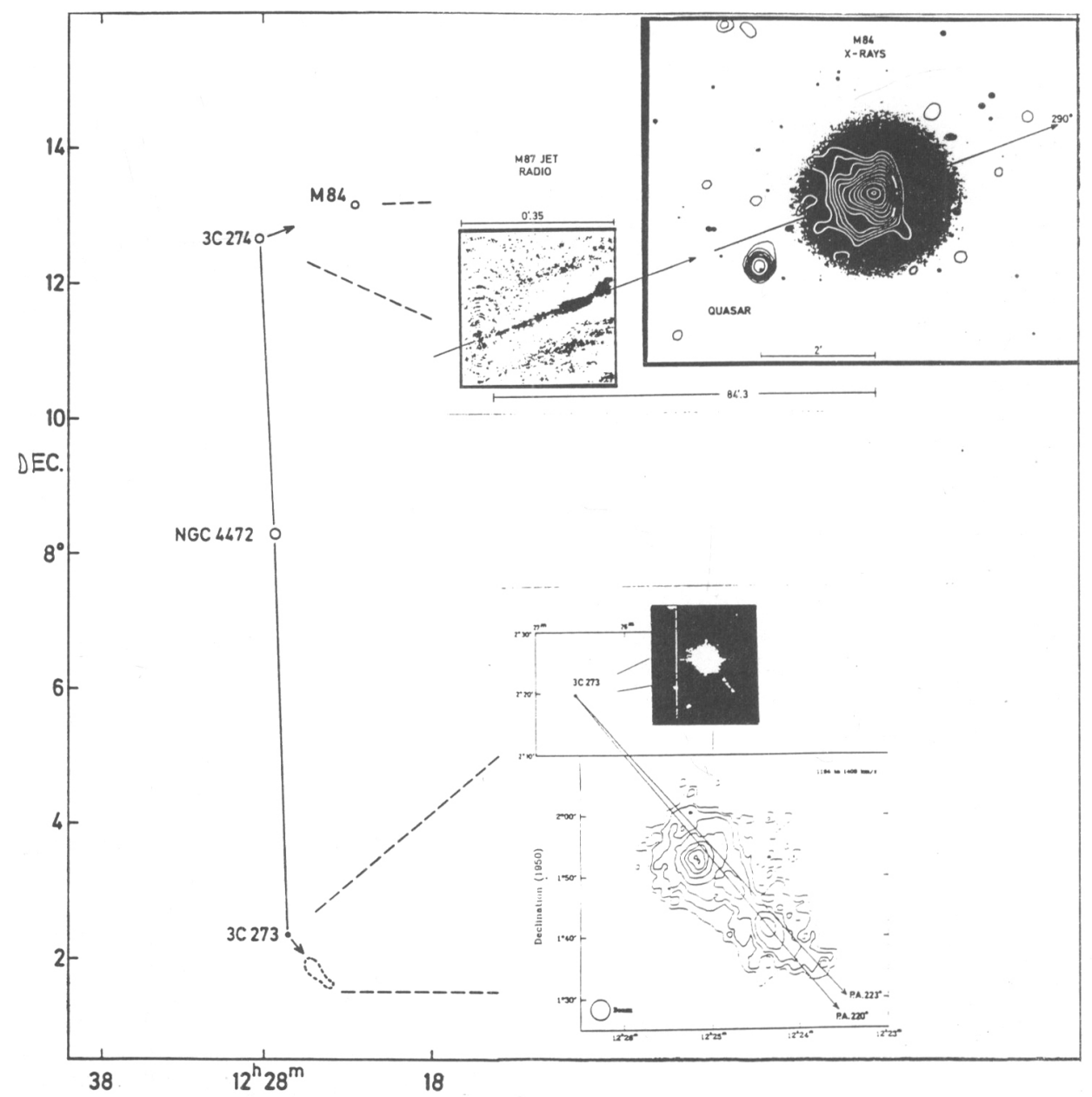

Fig. 3. Diagram showing the brightest galaxy in the Virgo Cluster, NGC 4472, with 3C273 and 9C274 aligned almost exactly across it. Directions for the unique jets in the latter objects are shown by arrows. Inserts show magnified views of what is in the direction of these jets. Aligned with the jet in 3 C273 is the mysterious hydrogen cloud discovered by Giovanelli and Haynes.

ejecting galaxies.

3C273 was one of those original radio sources discovered paired across a peculiar galaxy. But the galaxy, No.134 in the Atlas of Peculiar Galaxies, turned out to be an extraordinary galaxy. It was the brightest galaxy in the biggest most conspicuous aggregate of galaxies in the sky - the Virgo Cluster. On the other side of this central galaxy was another bright, famous radio source - M87! What a startling coincidence that two of the brightest radio sources in the sky, 3C273 and 3C274 (the radio name for M87), fell directly across the brightest galaxy in the Virgo Cluster!!

Fig. 3 here shows just how exactly the two radio sources are aligned across the central galaxy in the Virgo Cluster (NGC 4472). With an accuracy of alignment within 
\pm 0.2 arc min the chances of the configuration being accidental was less than one in ten thousand. But given the placement of M87 on one side of NGC 4474 what is the chance that the brightest apparent magnitude quasar in the whole sky would fall almost the same distance and almost exactly aligned on the other side? Forget it, the chance is vanishingly small.

So it was evident in 1966 that M87 had been ejected from NGC 4472 on one side and $3 \mathrm{C} 273$ has been ejected out the other side. But by further amazing coincidence, the two most conspicuous optical jets ever found, absolutely unique, belong to M87 and 3C273. They are both just about 20 arc sec long. This would seern to be clinching proof, in a case already proved, that there was a physical association amongst all these objects.

So it became clear that the two objects ejected from the original galaxy were each, themselves undergoing secondary ejection. What was being ejected? In the case of M87, Fig. 3 makes clear there is another, smaller, but still powerful radio galaxy to which the jet points exactly. This galaxy, M84, was recently measured to have X-ray material around it - and the streaming back of the X-ray material showed M84 to be travelling away from M87 just along the line of the latter's jet. [6]

What is being ejected along the line of the $3 \mathrm{C} 273$ jet? Until very recently nothing was known along this line. But suddenly originating from the New York Times were reports that an extraordinary hydrogen cloud had been found in the sky by Riccardo Giovanelli and Martha Haynes. It was so peculiar that it was excitedly speculated as possibly the first proto material ever found in the universe in the actual process of collapsing to form a galaxy. (Never mind that the Big Bang says all that was supposed to have happened 15 billion years ago.) But the position of this cloud accidentally became known to Geoffrey Burbidge and he immediately noted that it was only about a cloud diameter or $\sim 45$ arc min from $3 \mathrm{C} 273$. When I later glimpsed a map of the cloud I saw immediately that it pointed directly to $3 \mathrm{C} 273$. Absolutely the finishing blow was that the famous jet in $3 \mathrm{C} 273$ pointed right down the length of this cloud! $[7,8]$

There is a relatively bright quasar so close to M84 that there is a chance of only $2 \times 10^{-3}$ of its being accidental. [9] Moreover its outer X-ray isophotes point back toward M84. There are two small radio sources $\sim 8^{\prime}$ on either side of $3 \mathrm{C} 273$ aligned with the optical jet of $3 \mathrm{C} 273$ and the axis of the hydrogen cloud. [7]

In general there was strong evidence for association of quasars with the Virgo Cluster already in 1970 and the evidence has increased with time. Some highlights are: 1) the clustering of radio quasars of $z \sim 1$ around Virgo which in addition shows systematic Faraday rotation by the magnetic plasma in the cluster [10] 2) The clustering of bright objective prism quasars in Virgo particularly in the vicinity of the jet galaxy M87 [9] and 3) Sulentic in 1988 [11] showed the density on the sky of the classical quasars in the Palomar Bright Quasar Survey was $4-6$ times greater in the direction of the Virgo Local Supercluster than in other directions.

But then, every different morphological type of galaxy in the Virgo Cluster has its own systematic redshift despite the fact that they are all at the same distance from the observer. [12] Recently spectra in the ultraviolet with Hubble Space Telescope of 3C273 revealed 5 to 10 times as many absorbing clouds between the redshift of the quasar and the cluster than observed in other directions in the sky. Are absorbing clouds excessively piled up just accidently in the line of sight beyond the Virgo Cluster? And high redshift quasars? And $z \sim 1$ quasars? And PBQS bright quasars? And high redshift spiral galaxies? Perhaps it would be easier to accept the extensive statistical evidence from Burbidge et al. [13] that objects of different redshift are simply physically clustered together in space.

\section{The Introduction of X-Ray Evidence - Mark 205/NGC 4319 Updated}

One of the most famous examples of a quasar-like object apparently connected to a lower redshift galaxy is Mark 205, situated 40 arc sec south of the disrupted spiral galaxy NGC 4319. Following the verification of the optical connection [14], however, further progress was made very slowly. Jack Sulentic eventually managed to obtain time on the VLA radio telescope. His results are shown in Fig. 4 where the radio isophotes (in black) 
reveal an extremely rare event for a spiral galaxy - radio ejections emerging from the nucleus. Perhaps only in the rare spiral galaxy that is as torn apart as NGC 4319 can the radio ejections from the nucleus escape beyond the boundaries of the galaxy.

But the white isophotes show X-ray emission in the system. The recent accidental uncovering of this $\mathrm{X}$-ray emission in the archives of the Einstein laboratory raises certain questions. Special, high resolution (HRI) observations were made on 22 November 1978 and showed clear elongation of the strong X-rays around Mark 205 back toward the nucleus of NGC 4319. The result was never published and eleven years later during preparation for ROSAT proposals I stumbled across these observations [15]. Requests to the U.S. allocation committee for a relatively short 10 thousand second exposure to confirm this connection was turned down. Currently a proposal to make this crucial observation is before the German allocation committee.

It is interesting that here is another example of X-ray emitting matter associated with the apparent ejection of a young object from $\mathrm{Fig}$. 4 The quasar Mark 205, $40^{\prime \prime} S$ of the understand the nature of this X-ray material, disrupted galaxy NGC4319. Radio iso-

its relation to the radio continuum jets and phote black, $X$-ray, white.

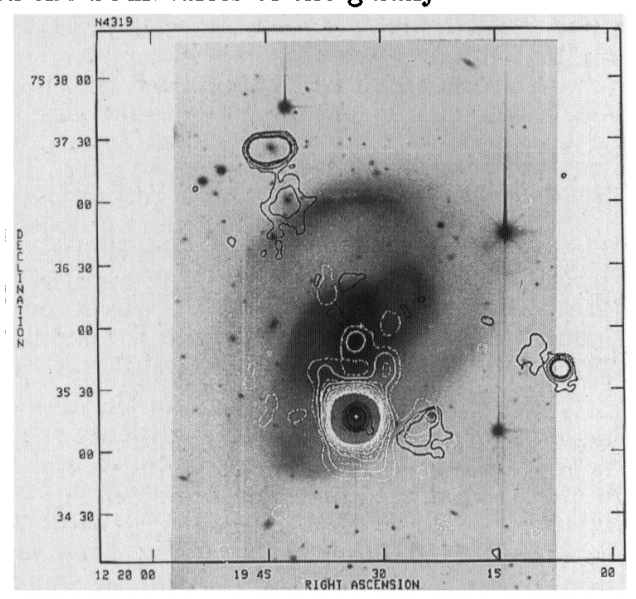

and its effect on the host galaxy. Optical studies involving high signal-to-noise observations of faint surface brightness features have been more or less pushed to present limits. The studies in other wavelengths such as X-ray, however, promise a bonanza of information, in objects such as this, on the physical nature of the ejections and their relation to the apparently associated higher redshift objects.

\section{The Statistics of Quasar/Galaxy Associations}

The most recent, and largest body of statistics arises from Burbidge et al. 's study of 577 QSO's closely paired with galaxies. [13] As many previous studies had found, there is an extremely significant excess of quasars close to lower redshift galaxies.

Fig. 5 proves in two ways that these are physical associations. First, at a given galaxy redshift the associated quasars cannot be accidental (because the area in which accidental discoveries can occur increases as the square of the angular separation but the observed quasars only occur close to the galaxy). Secondly, the observed separation of quasar from galaxy becomes larger as the redshift of the galaxy becomes smaller - that is, as the pair is closer to us in distance the apparent separation on the sky becomes larger. (see reference [14] for the most nearby associations which subtend large angles on the sky). 
Figure 5 also illustrates why gravitational lensing is not a viable explanation of the observations. If macro or micro lensing were responsible we should see the number of quasars building up toward the center of the galaxy until they were lost in the image of the galaxy (average limit indicated by lower hatched region in Fig. 5). Actually the quasars preferentially occur several diameter away from the galaxy. What can be out there to gravitationally lens background quasars?

Additionally it has now been realized that for gravitational lensing to account for excess numbers of quasars around galaxies that a steep increase of quasar numbers with fainter apparent magnitude would be required. The directly observed number of quasars shows far too slow an increase to be compatible with gravitational lensing. [16] It is instructive to note that the significance of these associations was originally rejected, then accepted by gravitational lens theorists and now once again ignored.

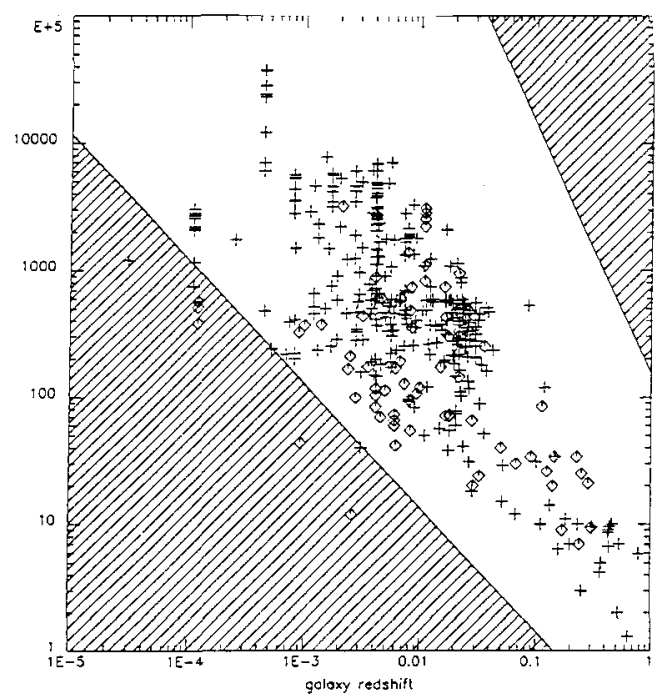

Fig. 5 Angular separation versus galaxy redshift for 392 galaxy/QSO pairs.

\section{Critical Cases of Quasars Associated with Galaxies}

Almost everyone of the 577 quasars paired with galaxies which were analyzed by Burbidge et al. [13] deserves deep, direct imaging study. But I mention a few here which could be especially rewarding.

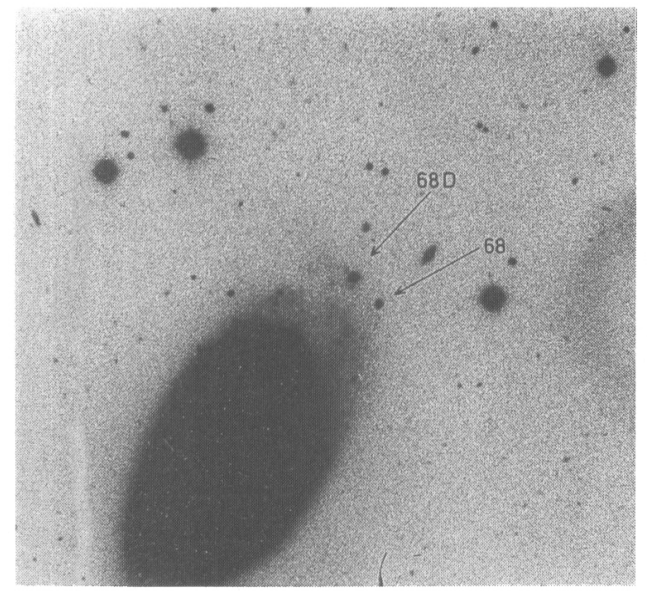

Fig. 6 Two high redshift quasars in the edge of the spiral galaxy NGC 470.

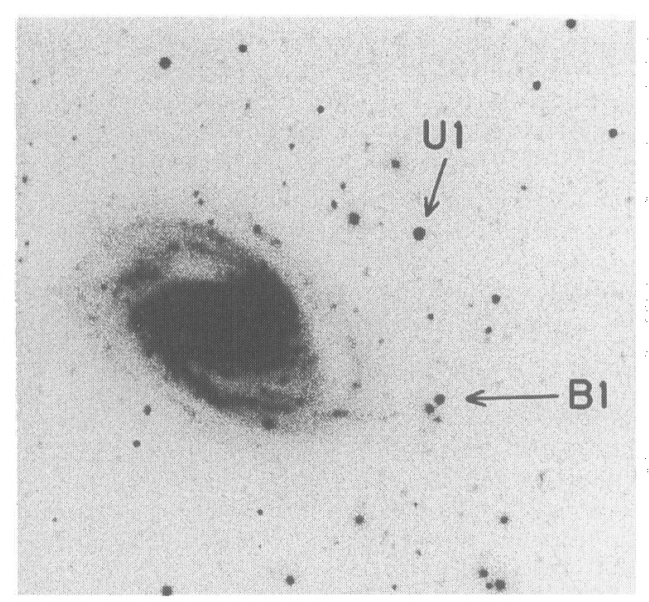

Fig. 7 Two quasars close to the spiral galaxy NGC 622.

Fig. 6 shows the bright spiral galaxy NGC 470 with quasars of redshift $z=1.88$ and 1.53 in its southeast edge. There is nebulosity with incipient structure apparently 
associated with the brighter quasar. This is extraordinary for a high redshift quasar. Is it interacting with the disk? The fainter quasar has suggestions of a long tail leading northward from it. The only deep photograph available is one made by Allan Sandage with the 200 -inch on a nearby ring galaxy on which NGC 470 was accidently registered. Another deep, good seeing direct image would be invaluable for confirmation of the NGC 470 features. But a better image than Fig. 6 could lead to sensational information on quasars and their relation to galaxies.

Fig. 7 shows another pair of quasars near a spiral galaxy. This case is particularly interesting because one straight spiral arm leads directly toward the quasar B1. But perhaps in the cases of both NGC 470 and NGC 622, high resolution radio measurements in both the hydrogen line and continuum would be even more informative than the optical investigations.

There are two famous cases of three quasars lying close to galaxies. (Accidental chances of $\lesssim 10^{-6}$ in both cases). In the one case, NGC 1073, photographs only by Hubble and a slightly better one by Sandage, are available. HI has been mapped in that spiral galaxy but no good X-ray maps are avallable. The other trio of quasars falls around the $E$ galaxy NGC 3842 which resides in a cluster of galaxies with an unusual number of discrete X-ray sources. Two of the three flanking quasars were discovered as X-ray sources. Like the quasar near M84 in the Virgo Cluster, the outer X-ray isophotes show distortions. Although considerable Einstein Observatory time was spent on this cluster better X-ray observations are today easily possible.

Other cases such as the four just mentioned are abundant and could give equally important results on further investigation.

\section{The Slowly Unfolding Puzzle of 3C 232/NGC 3067}

In 1971 Burbidge et al. [17] analyzed the known radio quasars and concluded they were associated with bright galaxies at a significance of $8.5 \sigma$. One of these, $3 \mathrm{C} 232$, had just by itself a chance of accidental association with the nearby NGC 3067 of only $2.5 \times 10^{-4}$. When it was discovered that absorption lines of low redshift hydrogen and calcium gas were seen in the quasar it was postulated that a large halo surrounded the galaxy through which a background quasar was being viewed. An equilibrium rotation curve was assumed for a supposed normal $\mathrm{Sb}$ galaxy and a hidden mass (dark matter) component was derived which was 16 times the visible mass of the galaxy! It mattered not that there was evidence available at the time that the galaxy was a peculiar, chaotic galaxy showing evidence for ejection? [18] But then 18 years elapsed before the hydrogen in NGC 3067 was mapped! (It was mapped for another reason). It was then discovered that an HI filament led directly from the galaxy to the quasar! At the point where it reaches the quasar the redshift dispersion of the hydrogen increases.

One puzzling result is that the hydrogen in the line of sight to the quasar is not excited to a state where it gives appreciable optical emission lines. (see Stocke et al. [19]). Does shift galaxy NGC 3067 . From the top, vithis mean that the quasar is several galaxy sual, hydrogen, $X$-ray.

diameters distant from the hydrogen filament? Or does it mean some kind of very narrow 
optical beaming for the QSO, or perhaps the continuum of the quasar should be observed further to the ultraviolet.

A wavelength region which could again give decisive information is the $\mathrm{X}$-ray. As the bottom frame in Fig. 8 shows, there is the possibility of an X-ray tail proceeding northward from 3C 232, away from NGC 3067 in a direction which would represent an extension of the hydrogen connection. The X-ray map is from a very short exposure which again lay unremarked in the Einstein archives. It would be extraordinarily simple and fruitful to check this apparent $\mathrm{X}$-ray connection with further observations.

\section{Again the Connection Between X-Rays, Ejection and Quasars}

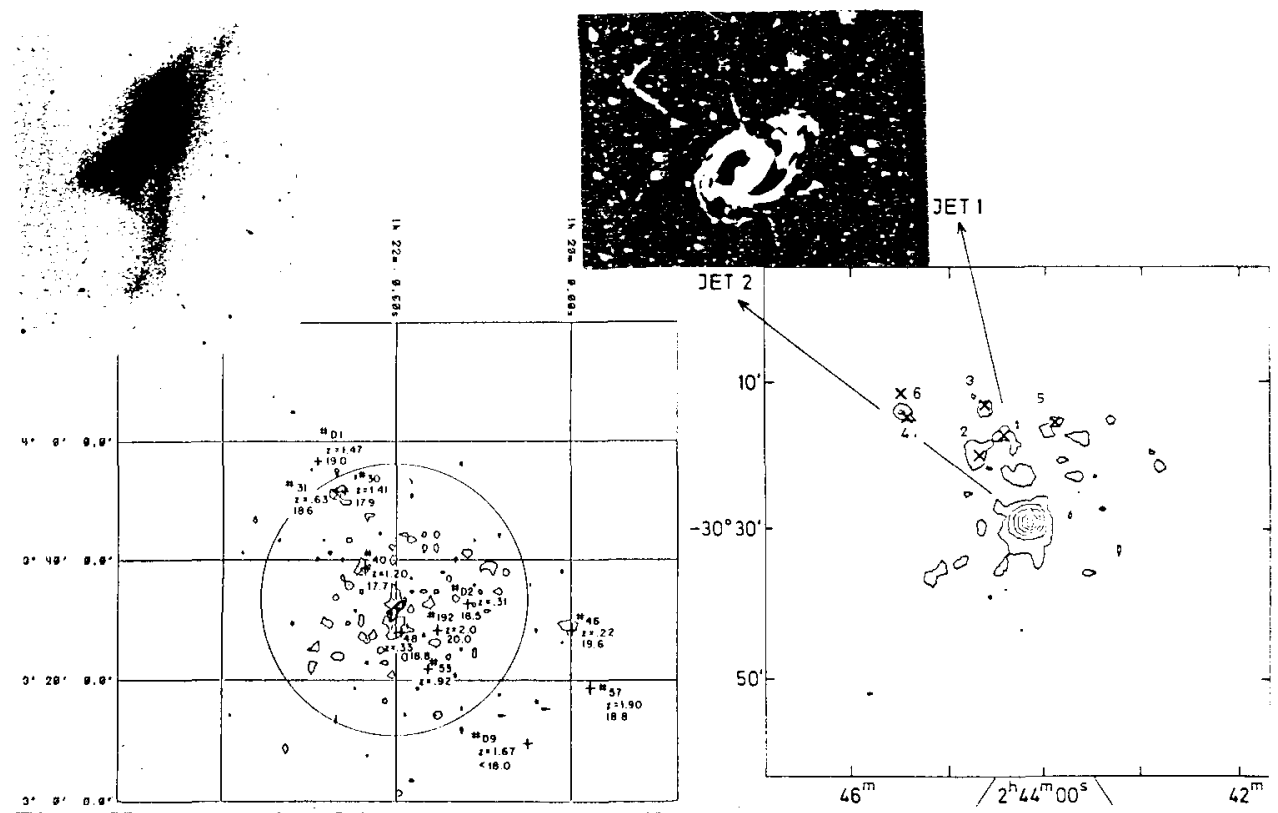

Fig. 9 NGC 520 visual image upper

left and $X$-ray map lower right.

Fig. 10 NGC 1097 with X-ray map lower right.

Two galaxies which are especially active and have many quasars associated with them are NGC 520 and NGC 1097.

a) NGC 520 is the brightest apparent magnitude, very disturbed galaxy in the sky after M82. In 1967 it was noticed that radio sources suggested ejection from it in a direction $\mathrm{NE}$ and SW. By 1970 a line of four radio bright quasars to the SW has been identified going accurately through the galaxy. In 1980 linitiated a search for more quasars around NGC 520. Astonishingly a line of 8 to 10 more quasars were revealed coming again NE and SW out of the disrupted galaxy! [14] In 1983 I ferreted out the fact that NGC 520 had been observed under the category of "normal galaxies" by the Einstein X-ray telescope. The X-ray map in Fig. 9 shows that the X-ray emission around NGC 520 breaks up into clouds and pieces many of which are identified with the quasars discovered in the complete quasar search around the galaxy.

b) NGC 1097 is a hot spot nucleus spiral galaxy with the longest, straightest optical jets known. In the direction of the stronger jets (NE) there are clouds and pieces of X-ray emission. Five of the six quasars in this region are identified with these X-ray patches. A careful search for optical quasars around NGC 1097 reveals a strong concentration of quasars centered on the galaxy. Within an area of 8.1 square degrees around NGC 1097 there are identified 30-40 quasars, the vast majority of which must belong to NGC 1097 . [14] 
The lesson taught by all the prior cases and which is so strongly reinforced by these last two cases, is that when there is strong evidence of ejection from a galaxy that there is usually $\mathrm{X}$-ray emitting material involved with this ejection.

This X-ray material is broken up into discrete, extended patches. Most of the quasars present coincide with the patches. The first order conclusion must be that the X-ray cloudlets are thrown out of the ejecting galaxies and therefore that the quasars, since the are identical to some of these patches, have also been ejected from the galaxies. The next question obviously is what is the generic relation of the X-ray material to the quasars and how do both result from the internal ejection processes in the nucleus of the galaxy.

These questions can be answered by faint surface brightness plus high resolution observations in optical, $\mathrm{X}$-ray and radio wavelengths. The instruments are available but the observations are not being made. The few investigations being carried out on these objects (for other purposes) can be easily named: Some guaranteed time observations by HST of the nucleus of NGC 1097, and long X-ray exposures with ROSAT of the optical jets in NGC 1097. The rest is free for observers with only modest aperture and modern detectors to work on.

\section{The Excess Redshift of Companion Galaxies}

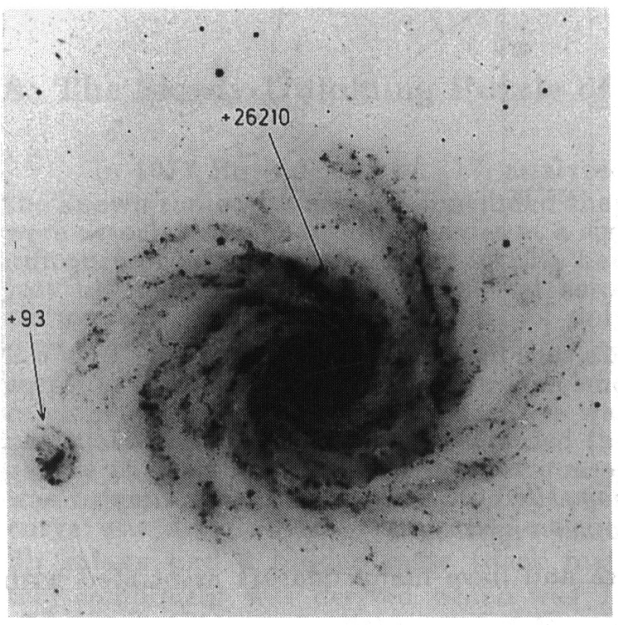

Fig. 11 NGC 1232 and companions

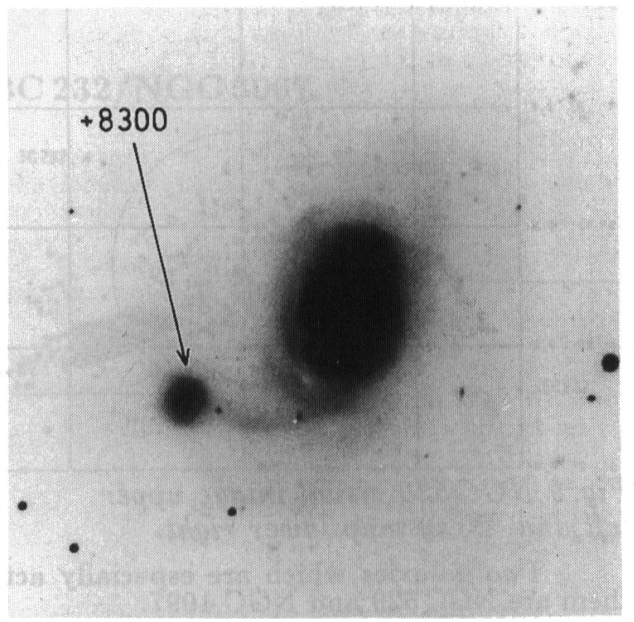

Fig. 12 NGC 7609 and companions

It has always been apparent that there is a continuity of physical relationships between quasars and galaxies. Starting with the point-like, active quasars we can trace a sequence of forms which progresses through compact galaxies, peculiar non-equilibrium galaxies, Sc spirals and then through the more relaxed Sb's, Sa's and E galaxies. [20] Their spectra too, show a continuous progression from high energy synchrotron through excited gas and young stars through galaxies dominated by quiescent old stars.

If quasars are companions to large, low redshift galaxies as we have seen, and they show excess relative redshifts, then it may not be so surprising that galaxies which are companions to large, low redshift galaxies also show excess redshifts. In fact they do. And, perhaps even more fascinating, the more they resemble quasars in compact morphology and active spectra, the higher their relative excess redshift.

This startling result surfaced in 1970 and continued to grow in significance until in 1982 a paper was published in $A p . J$. summarizing the result on 38 discordant redshift companions. [21] The list was restricted to cases where there was strong morphological evidence for each association. As a class of galaxies it was shown that they were excep- 
tionally rich in emission lines and characteristically showed early type absorption spectra. The excess redshift ranged from $4,600<c \Delta z<36,460 \mathrm{~km} \mathrm{~s}^{-1}$.

Two examples ar shown in Figs. 11 and 12. In the ScI galaxy NGC 1232, there is a Magellanic Cloud like companion at $+93 \mathrm{~km} \mathrm{~s}^{-1}$ and a very blue compact companion at $+26,210 \mathrm{~km} \mathrm{~s}^{-1}$ higher redshift than the main galaxy. In NGC 7603 the companion is $+8,300 \mathrm{~km} \mathrm{~s}^{-1}$ higher than the main galaxy which is a disturbed Seyfert type. These last two companions will be discussed later in connection with a possible explanation for their high redshift.

\subsection{NORMAL COMPANION GALAXIES}

We have considered quasar companions of low redshift galaxies which go from excess redshift of $.07<\Delta z \lesssim 2$. Next we have considered companion galaxies which have excess redshifts of $5,000<c \Delta z<36,000 \mathrm{~km} \mathrm{~s}^{-1}$. We noted that these latter companions are characteristically active, non equilibrium galaxies. Finally we now consider the most relaxed, most ordinary type companions.

The galaxies we know

the most about occur in the Local Group, of which our own Milky Way galaxy is a member, and in the nearby M81 group. Galaxies characteristically occur in groups and clus- $N$ ters in space and the M31 and M81 groups are typical of such associations. It is also characteristic of such groups that the largest, dominant galaxy in the group is of morphological type $\mathrm{Sb}$ and that their retinue of smaller companion galaxies runs the gamut of morphological type from irregular and $S c$ to dwarf $E$ galaxies. Fig. 13 shows how the dominant Sb's are always the lowest redshifts while the remaining, smaller companions have higher redshifts in the range $50<c \Delta z<300 \mathrm{~km} \mathrm{~s}^{-1}$. It should be emphasized that these are all the major com- Fig. 13 Relative redshifts in two nearest, major groups panions in these two well studied groups. The error bars on the points show that inaccuracies of measurement are negligible. All 21 out of the 21 major companions have positive redshift giving a chance of only one out of two million of happening accidentally if the effect were due to companions having plus and minus orbital velocities. Every test of additional groups at larger distances confirms the excess redshift of such companions. [22] We therefore have another extremely significant confirmation that companion objects to low redshift galaxies have significant positive redshift.

In the case of the galaxies represented in Fig. 13 we can ask, what property distinguishes the higher redshift companions from the lower redshift central galaxies. The answer is clear that on average the companions have earlier spectral type - indicative of a larger population of young stars. This can be seen easily in the spectra of the most extreme morphological cases such as NGC 404 and M82 where the spectra have early, A type absorption lines. Interestingly those two companions have the most extreme redshift discordances also. This correlation is shown very well by Girardi et al. who demonstrate for a different sample of companions that this excess redshift is correlated with the blueness of their color.

It is a reasonable inference that galaxies which are in non-equilibrium forms are young, because as time passes they would be expected to relax and become more regular. 
This applies especially to the very active, compact quasars. But as star formation proceeds in a galaxy the last sign of youth should be the early type, stellar absorption spectra. When these stars burn away they leave only the late spectral type stars in the old, relaxed galaxy. But it is just this sequence which correlates so well with the excess redshift as described in the preceding sections. The empirical conclusion must then be that companion objects to large, low redshift galaxies must be generally younger, and that the younger the companion the higher is its intrinsic redshift.

\section{Systematic Redshifts in OB Stars}

If companion galaxies have intrinsic redshifts then the stars in these galaxies must also have intrinsic redshifts because the stars in the mean give roughly the same redshift as the mean of the other constituents. In view of the preceding results it would therefore be reasonable to ask if younger stars have higher redshifts than older stars in a given galaxy. There are just a few external galaxies which are close enough so that we can test this proposal with accurate information on individual stars. Principal among there are the Magellanic Clouds (SMC and LMC). I take Hutching's 1980 data on the $34 \mathrm{OB}$ stars in the SMC and LMC as the most accurate available. [23] A simple count shows that $2 / 3$ of these young stars have higher redshift than the mean systematic redshift of their respective galaxies. This is a result at the $98 \%$ confidence level.

In order to check, however, that this result is not due to some systematic motion in $O B$ star atmospheres the mass loss indicators which were available for just these stars were examined. It turns out that corrections for mass loss did have to be applied but since they repre-

sent absorption lines coming toward the Fig. 14 Deviation from systemic redshift as a tions were positive and therefore the true function of mass-loss

$O B$ star redshift were even higher than actually measured.

Fig. 14 shows how the velocity of the $O B$ stars systematically decreases as the stellar wind blows harder. An accurate correction to the measured $O B$ star redshifts can be tabulated as a function of the number of its mass-loss indicators. When applied to the measured $O B$ star redshifts almost every OB star in the Magellanic Clouds (31 out of 34) turns out to be in excess of the systematic redshift of its galaxy. This is a result at the $6 \sigma$ level of significance. [24] Fig. 15 shows the distribution these OB star redshifts in the two Clouds.

The same mass-loss correction for measured redshifts is found for OB stars in our own galaxy. The result can be further checked by two early type supergiant objects in the nearby galaxy NGC 1569 and two young galaxies with unusually accurate absorption lines, NGC 2777 and NGC 4399. Again a minimum (before mass loss corrections) of $\geq 25$ to $\gtrsim 36 \mathrm{~km} \mathrm{~s}^{-1}$ is obtained for the mean excess of early type stars relative to the HI in each system. 
In the Magellanic Clouds, however, we can ask how young the individual stars are which give redshifts greater than the mean of the galaxy. Particularly in the SMC the stellar density is the lowest, which will minimize gravitationally caused velocity dispersion, and the absorption is a minimum so the absolute magnitudes of these stars can be derived most accurately. By putting the ten SMC supergiants in the Hertzsprung-Russell diagram (lu- No. minosity vs. temperature) we can interpolate between evolutionary tracks for stars of chemical composition similar to the SMC. It is a Fig. 15 The frequency distribution after correction for simple matter then to esti- mass-loss, of $34 O B$ stars with respect to the mean redshift mate the evolutionary ages of the Small and Large Magellanic Clouds of these ten
SMC. [22]

Fig. 16 shows that the evolutionary ages of the individual stars in the SMC are correlated with the amount of their excess redshift. After 25 years of seeing evidence for a general correlation of younger age versus higher intrinsic redshift it is a very exciting development to see an actually quantitative relation between these two parameters in extragalactic objects that we can study as closely as these individual stars can be studied.

\section{Numerical Calculations from an Redshift-Age Formula}

There is a necessary relationship between the redshift of matter and how much time has elapsed since it was created. This relation, which will be discussed in a later section is:

$$
\frac{1+z_{1}}{1+z_{0}}=\frac{t_{0}^{2}}{t_{1}^{2}}
$$

where $z_{0}$ is the redshift of matter created $t_{0}$ years ago and $z_{1}$ the redshift of matter created $t_{1}$ years ago. We take $t_{0}$ to be the age of the oldest created matter considered and, for reference, its redshift to be $z_{0} \equiv 0$. Then we ask how much later stellar matter would have to be created in order for that star to have an intrinsic, relative redshift of $z_{1}$. If the age of the oldest matter in its own reference frame is, for example, $\tau_{0}=17 \times 10^{9} \mathrm{yrs}$, then in our $t$ reference frame its age will be $3 \times \tau_{0}=t_{0}=51 \times 10^{9} \mathrm{yrs}$. (See section 14 for derivation of relation between $\tau$ time and $t$ time.) The following Table 1 gives sample values:

Table 1. Intrinsic redshift

- age calculations from (1)

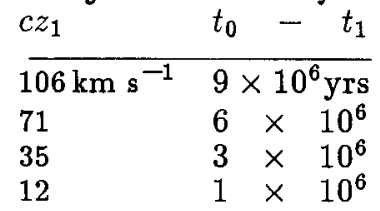


Figure 16 shows that if the youngest stars formed from first generation matter in the SMC are about $7-8 \times 10^{6}$ yrs evolutionary age, then all the stars of younger evolutionary age must come from matter created more recently. These later generation stars must have an intrinsic redshift - creation age envelope limit as shown by the dashed $c \Delta z$ line in Fig. 16.

It is an unexpected success of the theory as expressed in equation (1) to give quantitatively correct values for the size of the observed excess redshift and its dependence on age. It leads to a model which could explain the redshift anomalies which were the conclusion of the first part of this paper. This model is schematically represented in Fig. 17: A large $\mathrm{Sb}$ galaxy created $17 \times 10^{9} \mathrm{yrs}$ ago expels material which was formed $8 \times 10^{6} \mathrm{yrs}$ more recently. This companion will have an intrinsic redshift $\sim 100 \mathrm{~km} \mathrm{~s}^{-1}$ as the typical companion shown in Fig. 13. Material in the

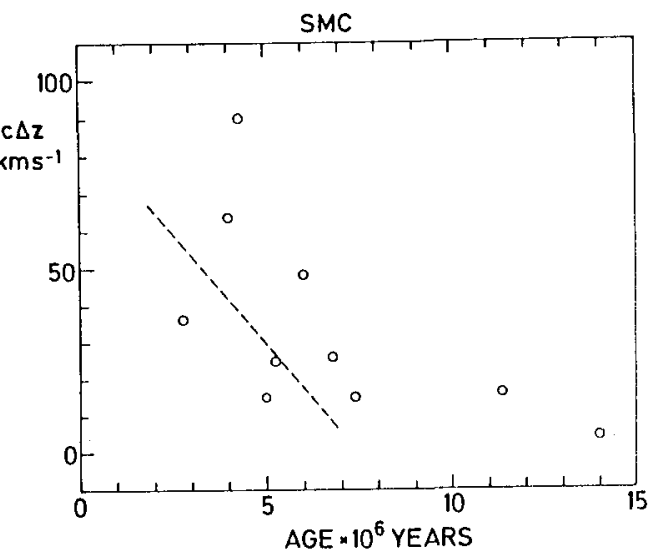
companion formed $3 \times 10^{6} \mathrm{yrs}$ later would Fig. 16 Excess redshifts as a function of form into stars with $\sim 35 \mathrm{~km} \mathrm{~s}^{-1}$ intrinsic evolutionary age in the $S M C$ redshift as observed in the SMC.

If this creation is pulsed in time then redshifts would come in mass discrete values in galaxies. Galaxy redshifts are indeed observed to come in discrete values and this quantization is otherwise inexplicable by current theory. It is important to note that age differences between epochs of creation of original and more recent matter are only a small percentage of the age of the oldest matter.

Even companion galaxies such

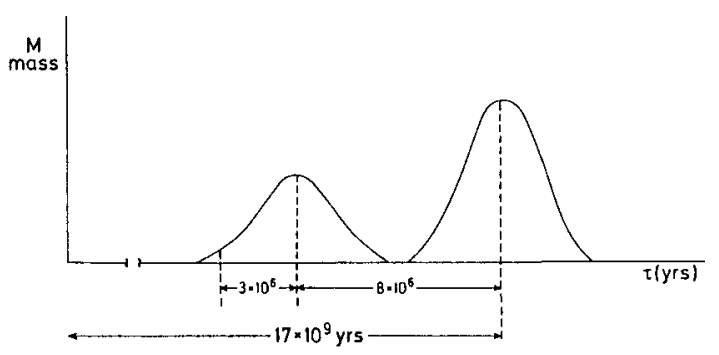

as NGC 7603 and NGC 1232 compact Fig. 17 Companion galaxy and the youngest stars blue companion, as discussed earlier in it forming from material $8 \times 10^{6}$ and $11 \times 10^{6}$ in section 10 , have ages which are younger than main galaxy

of the galaxies to which they are attached. The blue companion with an excess redshift of $z \sim 0.1 c$ is only $12 \%$ younger than its parent galaxy. This means that the integrated Hertzsprung-Russell diagrams of these higher redshift companions are very little different from those of the older galaxies. To detect this difference in the older stars of the $z \sim 0.1 \mathrm{c}$ companion it would be necessary to distinguish between stars 17 and $15 \times 10^{9} \mathrm{yrs}$ old.

Table 2. Intrinsic redshift - age calculation from (1)

Object $c z_{1}=c \Delta z \quad t_{0}-t_{1}=\Delta t$

NGC 7603 companion $8,000 \mathrm{~km} \mathrm{~s}^{-1} \quad 6.7 \times 10^{8} \mathrm{yrs.[22]}$

NGC 1232 blue companion $26,000 \quad 2.2 \times 10^{9} \quad[22]$

\section{The Hubble Relation as a Consequence of Look Back Time in a Static Universe}

There is one drastic result of eq.(1), however, and it can be seen already from Table 1 . If 
one looks at a galaxy $3.26 \times 10^{6}$ light years distant, (one Mpc) that galaxy is seen earlier in its history and its redshift is intrinsically $38 \mathrm{~km} \mathrm{~s}^{-1} \mathrm{Mpc}^{-1}$ higher. That is for an age of the oldest galaxies of $17 \times 10^{9} \mathrm{yrs}$. But if we take the age of the oldest galaxies to be $13<\tau_{0}<17 \times 10^{9} \mathrm{yrs}$ then the intrinsic redshift-distance relation comes out to be: $38<H_{0}<51 \mathrm{~km} \mathrm{~s}^{-1} \mathrm{Mpc}^{-1}$. In other words the Hubble constant predicted by eq. (1) is $H_{0}=45 \pm 7 \mathrm{~km} \mathrm{~s}^{-1} \mathrm{Mpc}^{-1}$. But the most recently measured $H_{0}=52 \pm 2 \mathrm{~km} \mathrm{~s}^{-1} \mathrm{Mpc}$. [25]

It is an astonishing fact that eq.(1), with only the age of the oldest stars as calculated from laboratory physics, gives directly the observed Hubble constant. In the universe then, for matter created at the same cosmological epochs there is a strict distance-redshift relation required, but very little distance-expansion velocity permitted, by the current measures.

This whole situation was anticipated by Fred Hoyle in 1972 when he produced the Hubble law in one mathematical step from an equation equivalent to (1). (see ref[22]) The only further step we have taken here is to assign a conventional age for the oldest galaxies which then yields a quantitatively correct Hubble constant.

Of course the age of the material in the systems which we are using for $z=0$ reference may in fact be older than $15 \times 10^{9} \mathrm{yrs}$. Then the $H_{0}$ predicted is less than $H_{0}=$ 45. That could leave room for some small expansion of the universe. Or, alternatively, the observed value $H_{0}$ may actually be smaller than $H_{0}=52$. Many values of $H_{0} \gtrsim$ $100 \mathrm{~km} \mathrm{~s}^{-1} \mathrm{Mpc}^{-1}$ have been measured and may simply reflect the inclusion of younger galaxies with intrinsic redshifts. For example if the smaller, luminosity weighted mean of Virgo Cluster redshifts is adopted [12], along with the Sandage-Tamann distance to Virgo of $21.9 \mathrm{Mpc}$, then an "observed" value of $H=47 \mathrm{~km} \mathrm{~s}^{-1} \mathrm{Mpc}^{-1}$ results. The few Sb and Sa galaxies in Virgo have negative redshifts and may represent slightly older epochs of material creation. We need to measure galaxies in Virgo (or anywhere) of a comparable creation age to our own to get the true value of $H_{0}$. The above estimates are probably representative of the uncertainties remaining.

\section{The Necessity of Non-Velocity Redshifts for More Recently Created Matter}

The basic reasoning with respect to the magnitude of mass of an elementary particle is that it must depend on the amount of material with which it can exchange gravitons. That in turn depends on the volume of the universe it sees, i.e. its light signal speed multiplied by the time during which it has been in existence.

The above reasoning is my interpretation of the theory of conformal gravity as developed by Fred Hoyle and Jayant Narlikar [26]. Formula (1) of the present paper is explicitly developed by Narlikar and Das [27] from calculations on the relative masses particles feel from the ratio of material within their light signal spheres. The energy of the photon an atom emits or absorbs (and hence inversely its redshift) is then proportional to the mass of the electron making the orbital transition.

The theory leads to the conclusion that elementary particles have masses which are a function of position and time, $m=m(x, t)$. It is important to stress that in the case where $m=$ constant, the theory reduces to the special case described by the accepted general relativistic formulation. The more general case where $m=m(t)$ is, however, required by the failure of the Big Bang theory and the arguments advanced for the continuous creation of galaxies. In the case of $m=m(t)$ the preceding paragraphs argue that redshift $z$, must then also be a function $z=z(t)$ of the epoch of creation of the matter.

Suggestions for the actual creation of matter go back to P.A.M. Dirac and later to Fred Hoyle's C (for creation) field in the cosmological equations. Dirac's multipicative creation would correspond here to creation of new matter in the nuclei of active galaxies. 
Its emergence would relate well to the evidence for the ejection origin of quasars and young galaxies.

\section{The Relation Between $t$ and $\tau$ time and Agreement with General Relativistic Solutions}

If we could watch the history of a nearby galaxy run backward in time we would see that masses of its constituent particles diminish as it approaches its origin. Now the rate at which atomic time runs $(\mathrm{R})$ is dependent on the mass of its particles $(m)$. Since $m$ was smaller in the past in this galaxy, its time appears to us to run slower. The amount of our time $(t)$ elapsed in an interval of the galaxy's time $(\tau)$ is:

$$
\frac{d \tau}{d t}=R(t) \alpha m \alpha t^{2}
$$

Then integrating the time difference backward to the origin:

$$
\tau=-\int_{t}^{0} \frac{t^{2}}{t_{0}^{2}} d t=\frac{t^{3}}{3 t_{0}^{2}}
$$

At the origin $t=t_{0}$ and $\tau=\tau_{0}$, giving:

$$
\tau_{0}=\frac{t_{0}}{3}
$$

This is the relation used in section 12 and thereafter. It simply means that viewed from our own reference frame, time in an early galaxy ran more slowly.

Differentiating equation (1) with respect to $\Delta t=t_{0}-t_{1}$ we obtain:

$$
\frac{d z}{d(\Delta t)}=\frac{2}{t_{0}}(1+z)^{3 / 2}
$$

In terms of the conventional expanding universe, $\Delta t=$ look back time to a galaxy at its inferred redshift distance and, for small $z, c z=v$. Therefore (5) becomes:

$$
\begin{gathered}
\frac{d c z}{d(c \Delta t)} \approx \frac{d v}{d r}=\frac{2}{t_{0}}(1+z)^{3 / 2}, \\
H=\frac{2}{t_{0}} \text { at } z=0,
\end{gathered}
$$

which is exactly what was found in section 13 for the Hubble relation predicted by equation (1) for small $z$.

It should be noted that for large $z$ the apparent expansion velocity in (6) is not constant but grows as $(1+z)^{3 / 2}$. This would predict that in a static universe where the redshifts were a function of look back time, that the apparent Hubble constant would grow larger with $z$. For $z=1.5$, for example $\frac{d z}{d \Delta t}=3.98 H_{0}$. If this were interpreted as a deviation from the Hubble relation in an expanding. universe it would require the galaxy to be 3 mag. more luminous in the past. These are just the order of deviations observed, 
but which are conventionally attributed to the systematic brightening of galaxies as we go back in time.

It is encouraging to note that the relations we have developed through elementary derivations from the principle that $m(t) \alpha t^{2}$, are all relations which come from a formal solution of the Einstein general relativistic equations for geometry and mass/energy:

$$
R_{\mu \nu}-\frac{1}{2} g_{\mu \nu} R=-8 \pi G T_{\mu \nu}
$$

The most commonly used solutions of these equations are the Friedmann models. Following Narlikar [28] these solutions can be transformed to flat space by making the coordinate transformation

$$
\tau=\frac{H^{2}}{12} t^{3} \quad \text { (we have reversed the } t, \tau \text { symbols used by Narlikar) }
$$

then at $\tau_{0}=t_{0} / 3$

$$
t_{0}=2 H^{-1} \text { and } \tau=\frac{2}{3} H^{-1} .
$$

These are the same as our derived relations (3), (4) and (7). We conclude therefore that we are using formally valid solutions of the basic general relativistic equations. We are simply given the quantities different interpretations. Namely, $m$ (const) $\rightarrow m(t)$ and $z(r) \rightarrow z(t)$.

\section{Solving Big Bang Problems by Reconsidering Discordant Redshifts}

The most difficult aspect of the observed associations between objects of much different redshift is that they appear to violate the redshift-distance rule. After all we do know that galaxies which are clearly like nearby ones, only seen at greater distances, do have systematically greater redshifts. The most important result of the age-redshift relation we have utilized here is that galaxies at the same evolutionary state must indeed manifest a strict redshift-distance relation. It just turns out that redshift does not mean velocity. Many scientists have suggested other interpretations of the redshift over the past 60 years. The velocity interpretation has always been the unprovable extrapolation.

To now have the observations interpreted consistently without expansion velocities rescues us from many paradoxes:

1) The quantization of galaxy redshift in units as small as $36 \mathrm{~km} \mathrm{~s}^{-1}$ has such high significance level $\left(10^{-4}<1-k_{0}<10^{-6}\right)$, globally [29], that very little real extragalactic velocity is permitted. Many observational results must currently be reconciled by the postulate of "hidden" or "dark" matter. This attempt to explain observational descrepancies with the theory cannot be verified by any current detections. Almost all of this hidden matter disappears - would simply be an incorrect interpretation - if redshifts have non-velocity components. Moreover the new theory suggests a simple physical mechanism for obtaining redshift quantization.

2) Younger, active objects such as quasars and early type companion galaxies can be at the same distance and can originate from larger, low redshift galaxies. This furnishes a natural evolutionary continuity in the morphology of extragalactic objects; young $\rightarrow$ old, active $\rightarrow$ relaxed, high $z \rightarrow$ low $z$. Continuous creation, the only alternative to the Big Bang, then is directly linked to a smooth scheme of development from matter $\rightarrow$ galaxies $\rightarrow$ stars.

This non-expanding universe which has been forced upon as by the detailed extragalactic observations then very simply reconciles the three current weaknesses in Big Bang theory which were discussed in the beginning of this paper: 
1) The smoothness of the cosmic background radiation can now be simply the radiation of the material vacuum, if that is the proper characterization of the intergalactic medium. It can come from regions local or distant because there is no velocity redshift to spread out the planck spectrum radiated from different distances.

2) There is no expansion age to the universe which is difficult to reconcile with the age of the oldest stars. The age of the oldest stars directly sets the redshift-distance relation, the so called Hubble constant. There are no adjustable parameters which can change this.

3) The existence of young galaxies which directly violate the Big Bang are then a natural consequence of continuous creation.

The continuous creation, static universe theory outlined above is, of course, only a theory. Many people in the past have had, and many will have in the future, contending theories. A theory after all is only the most consistent way of connecting all the known facts together. If the one suggested here fails or a better one comes along at least its enunciation will make, I hope, one important point. That point is that there is a theory that can fit the present observations - one should never discard the observations because they do not fit a theory. The observations described here are valid and represent an unmatched opportunity to gain better understanding of the universe.

\section{Some Further Examples of Strange Observations}

As an illustration of the moral we tried to draw at the conclusion of the last section let me show some observations which appear ludicrous on conventional assumptions. In Fig. 18 are shown all the galaxies (now quite completely known) with redshifts $300<$ $c z_{0}<700 \mathrm{~km} \mathrm{~s}^{-1}$ in the direction of the center of the Local Group of galaxies (M31 et al. ). The Local Group is not supposed to contain galaxies with redshifts in excess of about $100 \mathrm{~km} \mathrm{~s}^{-1}$. (Although it is not clear why more distant groups are conventionally permitted to have much higher redshift dispersions). In any case the Local Group appears to contain a line of galaxies which have redshifts of up to $700 \mathrm{~km} \mathrm{~s}^{-1}$. That line goes through the center of M31. Also in that same line are the conventional dwarf companions of M31 plus M33 and NGC 404. But even more startling are three negative redshift $\mathrm{HI}$ clouds and a line of apparent galactic cirrus going down to NGC 918, which appears to interacting with the cirrus. [30] The line then continues towards $3 \mathrm{C} 120$ which as we saw in section 3.0 of this paper, has $F i g .18$ All galaxies with $300 \leq c z_{0} \leq 700 \mathrm{~km} \mathrm{~s}^{-1}$

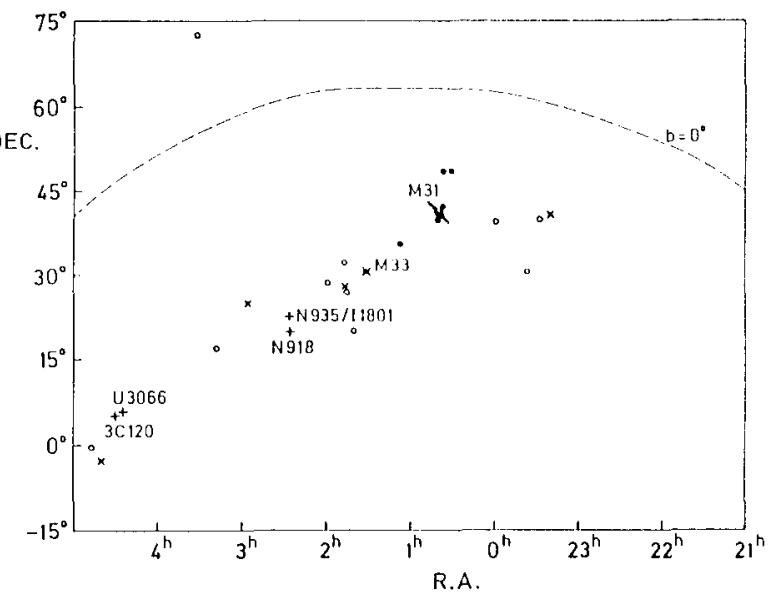
galaxies and quasars clustered a- plus Local Group galaxies, $-86 \leq c z_{0} \leq 142 \mathrm{~km} \mathrm{~s}^{-1}$ round it. Most recently large, $100 \mu$

infrared clouds have been reported emerging from either side of $3 \mathrm{C} 120$ and its neighboring galaxy NGC 3066. [31]

If we did not have the Burbidge et al. result [13] that objects cluster together regardless of redshift we should certainly be tempted to ignore these observational facts. But now if we also have the working hypothesis that objects of different ages can have different redshifts we see that objects from the redshift of the HI clouds $\left(c z_{0}=-130,-140 \mathrm{~km} \mathrm{~s}^{-1}\right)$ 
to the redshift of $3 C 120\left(c z_{0}=10,000 \mathrm{~km} \mathrm{~s}^{-1}\right)$ can be encompassed by ages which are only a small percentage different from the age of M31. If this pattern is repeated perhaps we should take the phenomenon seriously.

Fig. 19 shows all the galaxies with $3100<c z_{0}<$ $5100 \mathrm{~km} \mathrm{~s}^{-1}$ plotted over a large region of the sky. They appear to run on a line through $\mathrm{M} 81$ as a center. In order to test whether this is a signifi- $03700-5100 \mathrm{k} / \mathrm{s}$ cant physical phenomenon the $\cdot 3100-3700$ 20 brightest apparent magnitude spiral galaxies north of Dec $=0^{\circ}$ were examined. Of the 14 which are uncrowded by nearby bright galaxies a total of 13 have wehl marked lines and concentrations of fainter, higher redshift galaxies [30]. Can this be true? We have to honor the observations. The next, perhaps quite rewarding

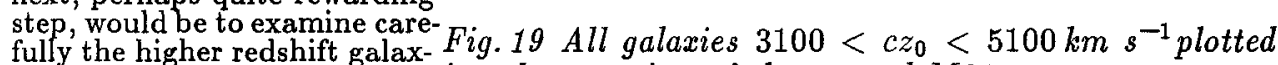
ies in these lines. Are there in a large region of sky around $M 81$ differences between them and the large low redshift galaxy which could be manifestation of lower luminosity?

\section{References}

1. Arp, H., Burbidge, G., Hoyle, F., Narlikar, J.V. and Wickramasinghe, N.C. (1990) 'The Extragalactic Universe an Alternative View', Nature 346, 807.

2. van den Bergh, S. (1990) 'Cosmology - In Search of a New Paradigm', J. Roy. Astr. Soc. Can. 84, 275.

3. Arp, H. (1990) 'Galaxy Creation in a Non-Big Bang Universe', Third Philosophy and Physics Workshop of the Forschungsstätte der Evangelischen Studentengemeinschaft.

4. Baldwin, J.A., Carswell, R.F., Wampler, E.J., Smith, H.E., Burbidge, E.M., Boksenberg, A. (1980) 'The Nebulosity Associated With 3C 120', Ap.J. 236, 388.

5. Arp, H. (1987) "3C 120 and the Surrounding Region of Sky', J. Astrophys. Astr. (India) 8, 231.

6. Arp, H. (1986) 'Relation of the Jet in M87 to Nearby Galaxies in the Virgo Cluster', $J$. Astrophys. Astr. (India) 7, 71.

7. Arp, H. and Burbidge, G. (1990) 'The Peculiar Hydrogen Cloud in the Virgo Cluster and 3C 273', Ap.J. 359, L1.

8. Arp, H. (1990) '3C 273 in Perspective', Astronomy Now 4, 43.

9. Arp, H. (1990) 'Quasars in the Central Region of the Virgo Cluster', J. Astrophys. Astr. (India) 7, 71 .

10. Arp, H. (1988) 'Intergalactic Magnetic Field and the Distance of Quasars from Faraday Rotation', Physics Letters A 129, 135.

11. Sulentic, J.W. (1988) 'An Apparant Correlation Between the Brightest Galaxies and Quasars', Physics Letters $A$ 131, 227.

12. Arp, H. (1988) 'What is the Mean Redshift of the Virgo Cluster?', Astron. Astrophys. 202,70 .

13. Burbidge, G., Hewitt, A., Narlikar, J.V. and Das Gupta, P. (1990) 'Associations Be- 
tween Quasi-Stellar Objects and Galaxies', Ap.J. 74, 675.

14. Arp, H. (1987) 'Quasars, Redshifts and Controversies', Interstellar Media, Berkeley.

15. Arp, H. (1990) 'X-Ray Connection From the Quasar Markarian 205 to the Galaxy NGC 4319', Physics Letters A 146, 172.

16. Arp, H. (1990) 'Quasar Numbers as a Function of Apparent Magnitude: Gravitational Lensing of Quasars Associated with Nearby Galaxies?', Astron. Astrophys. 229, 93.

17. Burbidge, E.M., Burbidge, G.R., Solomon, P.M. and Strittmatter, P.A. (1971) 'Apparent Associations Between Bright Galaxies and Quasi-Stellar Objects', Ap.J. 170, 233.

18. Arp, H. (1989) 'The HI Bridge Between NGC 3067 and 3C 232' ESO Workshop on Extranuclear Activity in Galaxies, ESO Conf. Proc. No. 32, p.89

19. Stocke, J.T., Case, J., Donahue, M., Skull, J.M. and Snow, T.F. (1991) 'New Observations on the Gas Cloud Associated with the Quasar-Galaxy Pair 3C 232/ NGC 3067', Ap.J. 374, 73.

20. Arp, H. (1976) Anomalous Redshifts in Galaxies and Quasars, I.A.U. Colloque No. 37, 'Decalage Vers Le Rouge et Expansion De L'Universe' Edition Du CNRS, PARIS, p.377.

21. Arp, H. (1982) 'Further Examples of Companion Galaxies with Discordant Redshifts and their Spectral Peculiarities', Ap.J. 263, 54.

22. Arp, H. (1991) 'How Non-Velocity Redshifts in Galaxies Depend on Epoch of Creation', APEIRON No. 9-10, 18.

23. Hutchings, J.B. (1980) 'Stellar Winds from Hot Stars in the Magellanic Cloud', Ap.J. $235,413$.

24. Arp, H. (1991) 'Systematic Redshifts in OB Stars', Astron. Astrophys. (submitted).

25. Sandage, A. and Tamman, G.A. (1990) 'The Cosmic Value of $H_{0}$ Freed from All Local Velocity Anomalies', ESO Preprint No. 722.

26. Hoyle, F. and Narlikar, J. (1974) 'Action at a Distance in Physics and Cosmology' (San Francisco: Freeman)

27. Narlikar, J. and Das, P.K. (1980) 'Anomalous Redshifts of Quasi-Stellar Objects', Ap.J. 240, 401.

28. Narlikar, J. (1977) 'Two Astrophysical Applications of Conformal Gravity', Ann. of Physics 107, 325.

29. Napier, W.M. (1991) 'Crystal Spheres in Velocity Spaces?', APEIRON No. 9, 8.

30. Arp, H. (1990) 'Galaxy Alignments', J. Astrophys. Astr. (India) 11, 411.

31. Arp, H. (1991) 'The Probable Extragalactic Nature of Some Low Surface Brightness Clouds at High Galactic Latitude', Astrophys. and Space Sci., in press. 